INPLASY

PROTOCOL

To cite: Xu et al. A Meta analysis of ablation indexguided pulmonary vein isolation. Inplasy protocol 202060074. doi: 10.37766/inplasy2020.6.0074

Received: 20 June 2020

Published: 20 June 2020

Corresponding author: Xu Min

286412861@qq.com

Author Affiliation:

Mianyang 404 Hospital

Support: Health Commission of Sichuan $\mathbf{P}$

Review Stage at time of this submission: The review has not yet started.

Conflicts of interest:

The authors have no conflicts of interest to declare.

\section{A Meta analysis of ablation index-guided pulmonary vein isolation}

Xu, M1; Yang, Y2; Zhang, D³.

Review question / Objective: Could Al guided PVI stratagy(based on CARTO 3 V4, Biosense Webster, Inc., Diamond Bar, CA) improve outcomes in patients with Paroxysmal AF or Persistent AF after PVI compare with tranditional index guided?

Condition being studied: Pulmonary vein potential is one of the major triggering foci in occurence of atrial fibrillation(AF). Pulmonary vein isolation(PVI) has become the basic procedure for radiofrequency ablation. Durability of pulmonary vein isolation is associated with recurrence of atrial fibrillation after radiofrequency ablation. It is important to impove Index guided PVI to reduce the occurrence of pulmonary vein reconnection. Ablation Index is a novel paramater in evaluating the depth of ablation.It was based on the CARTO system, which integrated conventional index such as pressure and energy, and could be used to guide Ablation depth and reliability in real-time during surgery. At present, a small number of non-randomized studies suggest that Alguided PVI could improve the clinical outcome of pulmonary vein isolation. But so far, there is insufficient evidence-based medical evidence to support this view.We did this study to evaluate the efficiency and safety of Al guided PVI and hope to provide new evidence for this new strategy.

INPLASY registration number: This protocol was registered with the International Platform of Registered Systematic Review and Meta-Analysis Protocols (INPLASY) on 20 June 2020 and was last updated on 20 June 2020 (registration number INPLASY202060074).

\section{INTRODUCTION}

Review question / Objective: Could Al guided PVI stratagy(based on CARTO 3 V4, Biosense Webster, Inc., Diamond Bar, CA) improve outcomes in patients with
Paroxysmal AF or Persistent AF after PVI compare with tranditional index guided?

Rationale: PVI is the basic procedure of ablation for Atrial fibrillation. Improving the wall penetration effectiveness and uniformity of point-to-point ablation is a hot 
research topic. In recent years, many nonRCT studies suggested that Al guidance can improve the clinical effeciency of PVI, but there is no enough evidence at present.

Condition being studied: Pulmonary vein potential is one of the major triggering foci in occurence of atrial fibrillation(AF). Pulmonary vein isolation(PVI) has become the basic procedure for radiofrequency ablation. Durability of pulmonary vein isolation is associated with recurrence of atrial fibrillation after radiofrequency ablation. It is important to impove Index guided PVI to reduce the occurrence of pulmonary vein reconnection. Ablation Index is a novel paramater in evaluating the depth of ablation.lt was based on the CARTO system, which integrated conventional index such as pressure and energy, and could be used to guide Ablation depth and reliability in real-time during surgery. At present, a small number of non-randomized studies suggest that Alguided PVI could improve the clinical outcome of pulmonary vein isolation. But so far, there is insufficient evidence-based medical evidence to support this view.We did this study to evaluate the efficiency and safety of Al guided PVI and hope to provide new evidence for this new strategy.

\section{METHODS}

Search strategy: The databases included PubMed, Cochrane, EMbase, ScienceDirect and the chinese medical database,such as Wanfang database, China National Knowledge Infrastructure (CNKI), CQVIP, CBM library. The language was limited to English or Chinese, and the retrieval time was from 2015 to the start of this study. Boolean logic operators (AND, OR, NOT) were used to search for keywords and free words. The keywords included "Ablation index", "Atrial Fibrillation", "Catheter Ablation", "Radiofrequency ablation", etc.

Participant or population: Non-valvular patients diagnosed with paroxysmal or persistent atrial fibrillation.Regardless of prior radiofrequency ablation history or not.
Intervention: Ablation index guided pulmonary vein isolation.

Comparator: Traditional index(Contact force,impedance drop,et al) guided pulmonary vein isolation.

Study designs to be included: Clinical studies about Al-guided PVI(supported by the VISITAGTM module of the CARTO3 system) written in Chinese and English, disregard for retrospecti.

Eligibility criteria: 1) Subjects: (1) paroxysmal AF or persistent AF; (2) Alguided PVI supported by the VISITAGTM module of the CARTO3 system (CARTO®3 V4, Biosense Webster, Inc., Diamond Bar, CA). (2) Published within the past 5 years. (3) public publication.

Information sources: PubMed, Cochrane, EMbase, ScienceDirect and the chinese medical database,such as Wanfang database, China National Knowledge Infrastructure (CNKI), CQVIP, CBM library.

Main outcome(s): Intraoperative and postoperative indicators, e.g. The first-pass PVI,The long-term arrhythmia-free survival,Complications, Fluoroscope time, ablation time, and procedure time, and so on.

Quality assessment / Risk of bias analysis: The Newcastle -Ottawa scale for the observational studies.

Strategy of data synthesis: Statistical analysis was performed using RevMan version 5.3 software (The Cochrane Collaboration). Standardized mean difference was used to combine statistics for continuous variables, with the relative risk (RR) and 95\% confidence interval (Cl) as the collection metrics for dichotomous data $P<0.05$ indicated that the difference between the two groups was statistically significant. The fixed-effect model (FEM) was used when $12<50 \%$ or $P>0.05$ in the homogeneity test, and the random-effects model (REM) was used when $12>50 \%$ or $P$ $<0.05$ in the homogeneity test. As for the 
combined effect of the included studies, $P$ $<0.05$ indicated that the difference was statistically significant, and $P<0.01$ indicated that the difference was extremely significant.

Subgroup analysis: 1) patient heterogeneity, 2) different duration of follow-up, 3) Data heterogeneity and cause analysis. If it cannot be quantified, it will be presented as a narrative.

Sensibility analysis: Heterogeneity will be assessed statistically using the sensitivity analysis.

Language: The language was limited to English or Chinese.

Country(ies) involved: China.

Keywords: atrial fibrillation; radiofrequency ablation; pulmonary vein isolation; ablation index; meta-analysis.

Contributions of each author:

Author 1 - Min Xu - The author drafted the manuscript.

Author 2 - Yan Yang - The author was responsible data extraction and quality assessment.

Author 3 - Dayong Zhang - The author was responsible data extraction and quality assessment. 